\title{
Aportaciones de la formación blended learning al desarrollo profesional docente
}

\section{Contributions of blended learning training to teacher professional development}

\author{
Ana Duarte Hueros \\ María Dolores Guzmán Franco \\ Universidad de Huelva (España) \\ Carmen Yot Domínguez \\ Universidad de Sevilla (España)
}

\section{Resumen}

El eje vertebrador de este estudio es el análisis de la implementación de una metodología docente combinada (presencial y virtual o blended learning) de forma equilibrada e integrada en la formación del profesorado y la importancia del liderazgo para planificar, atender y coordinar este proceso. Comenzamos realizando una revisión sistemática de la literatura de investigación de los últimos quince años (2002 al 2017) en la base de datos principal de Web of Science (WOS) considerada la plataforma de mayor prestigio entre la comunidad científica, identificándose 190 estudios relacionados con los tópicos: Blended learning, desarrollo profesional docente y liderazgo educativo. De ellos se seleccionaron aquellos que se adscriben a la categoría investigación educativa (education educational research), localizándose 163 documentos, de los cuales, 75 son artículos. Por último, se afinó la búsqueda excluyendo aquellos artículos que se relacionaban con otras áreas de investigación, ya que nuestro centro de interés era el desarrollo profesional del docente, encontrando un total de 35 títulos que cumplían todos los criterios preliminares. La muestra resultante fue de 24 artículos que reunían todas las características que nos habíamos planteado. Los resultados reflejan que el blended learning es valorado como un modelo de formación que permite la adquisición de competencias, puede favorecer el desarrollo profesional, fomentar el trabajo colaborativo, aumentar las destrezas técnicas y didácticas del profesorado hacia la tecnología, promover experiencias interdisciplinares, compartir innovaciones, etc., entre otras posibilidades.

Palabras clave: desarrollo profesional; blended learning; competencias; liderazgo; formación.

\begin{abstract}
The central theme of this study is the analysis of a balanced integrated teaching methodology (face-to-face and virtual, as blended learning) and the extent of its implementation in teacher training, as well as the importance of leadership in planning, supervising and coordinating this process. We began with a systematic review of the literature of the last 15 years (2002-2017)
\end{abstract}


on the Web of Science (WOS), the most highly rated database in the scientific community. We identified 190 studies related to blended learning, professional teaching development and leadership in education. We then selected 163 documents that fell specifically into the educational research category, of which 75 were articles. We further fine-tuned the search by excluding those articles related to research fields other than teachers' professional development, and arrived at 35 articles that fulfilled our preliminary criteria. We reduced the sample to the 24 articles that contained all the features required by our investigation. The results show that blended learning is a valuable training tool that enables teachers to acquire competences and which can aid their professional development; it can also foment collaborative work, augment teachers' technical and didactic skills around technology, promote interdisciplinary experiences and help teachers to share innovations, etc., among other potential outcomes.

Keywords: professional development; blended learning; competences; leadership; tranining.

Cada vez aparecen más estudios que demuestran la importancia que tiene el desarrollo profesional docente y el liderazgo en los procesos formativos y organizativos que se producen en contextos educativos, ejerciendo una poderosa influencia en la planificación, desarrollo y evaluación, en la eficacia de las tareas técnicas propias de la gestión y en la calidad del sistema relacional del mismo. Por ello, cuando se tratan aspectos relacionados con el liderazgo no se refiere a algo periférico o meramente teórico, sino que se trata de un tema clave en el contexto educativo (Leithwood y Louis, 2011; Argos y Ezquerra, 2013; Bolívar, López y Murillo, 2013).

Queremos resaltar la importancia del desarrollo de competencias vinculadas al liderazgo educativo. De hecho, al discurrir las tareas del líder entre las actividades formativas y organizativas, el rol se convierte en una compleja labor, ya que no es una tarea fácil conseguir una buena armonía entre un perfil de gestión y liderazgo que sea capaz de coordinar, motivar, administrar autoridad, y que al mismo tiempo, sea capaz de dinamizar en equipo un proceso de diseño, ejecución y evaluación de Proyectos, elaborados consensuadamente. El responsable de liderar, ya sea en un aula, en un centro o en un entorno telemático, debe encontrar equilibrios, tiene que compartir roles, planificar, consensuar y desarrollar tareas. Para comprender el trabajo de las personas que asumen liderazgo es necesario una visión integral y entender que las múltiples dimensiones implicadas en su labor están conexionadas.

En el desarrollo profesional docente, las cuestiones relacionadas con el liderazgo educativo, pueden asociarse a una interesante línea de actuación específicamente relacionada con los planes de formación inicial y programas de formación continua. Línea de desarrollo docente vinculada no solo al aprendizaje formal, sino también al aprendizaje informal y no formal, que permita al profesional conectar el conocimiento necesario con las experiencias individuales y las prácticas colectivas, es decir, contextualizar el conocimiento que necesita en la práctica generando una reflexión compartida y significativa (Bernal e Ibarrola, 2015). 
El liderazgo, pues, se trata de una función que incide en todo el proceso organizativo y formativo, distribuyéndose por todas las unidades de la misma y constituyendo uno de los valores que integran la cultura y el contexto; y además, en su desarrollo influyen factores personales y profesionales.

La integración de las TIC y sus posibilidades para la formación y la organización, suma funciones a las desarrolladas tradicionalmente por los líderes en el ámbito de la educación, pudiendo llegar a convertirse en el impulsor y coordinador de proyectos que combinen entornos tradicionales con nuevos escenarios de aprendizaje.

La Ley Orgánica 8/2013, de 9 de diciembre, para la Mejora Educativa (LOMCE) hace especial hincapié en este aspecto ya desde su preámbulo, pues afirma que las Tecnologías de la Información y la Comunicación serán una pieza fundamental para producir el cambio metodológico que conduzca a la consecución del objetivo de mejora de la calidad educativa, así como en la formación del profesorado y en el aprendizaje de los ciudadanos a lo largo de la vida.

El Marco Común de la Competencia Digital Docente, vigente desde 2012, adaptado del Marco europeo DIGComp, actualizado por JCR en 2016 y coordinado por INTEF (Instituto Nacional de Tecnologías Educativas y de Formación del Profesorado) del Ministerio de Educación, Cultura y Deportes, en su última versión publicada en 2017 describe de manera estandarizada la competencia digital de los docentes en cinco áreas: Información y alfabetización informacional, Comunicación y colaboración, Creación de contenido digital, Seguridad y Resolución de problemas; apostando por la formación del profesorado en competencias digitales como factor clave en el desarrollo profesional.

En el contexto universitario, desde comienzos de siglo, la Ley Orgánica 6/2001, de 21 de diciembre, de Universidades se marcaba como objetivo responder a los retos derivados de la enseñanza superior no presencial a través de las nuevas tecnologías de la información y la comunicación y abrir nuevas posibilidades a la formación.

Desde 2007 a 2016, la CRUE-TIC edita anualmente el Informe UniversiTIC, que publica la Comisión Sectorial de Tecnologías de la Información y la Comunicación de las Universidades españolas y aportan un estudio sobre la integración y posibilidades de las TIC en el contexto universitario y las posibilidades que estas brindan al futuro desarrollo profesional claramente marcado por el uso de las TIC y el incremento del blended learning, dato coincidente con el Informe Horizon 2016 que destaca que una de las tendencias con mayor impacto a corto plazo en la educación será la generalización de un tipo de aprendizaje mixto o híbrido (blended learning).

\section{OBJETIVOS}

El objetivo general que nos propusimos con este estudio fue el de realizar una revisión sistemática de los estudios de investigación 1) que han abordado la aportación que el blended learning como modalidad de formación hace al desarrollo profesional docente, 2) sobre los que se ha estado trabajando en los últimos quince 
años y 3) cuyos resultados ya han sido dados a conocer puesto que se han publicado en revistas científicas de impacto. Así, las preguntas de investigación que nos guían en el esfuerzo de búsqueda y análisis de los trabajos son las concernientes a: ¿̇cuántos estudios de investigación se han preocupado por la formación blended learning para el desarrollo profesional de los docentes?, ¿cómo podemos caracterizar a estos estudios (en base a su objeto de estudio, su metodología, etc.)? y ¿̇qué evidencias se han producido a partir de ellos sobre la contribución que el blended learning hace al desarrollo profesional de los docentes?

En la pretensión de aproximarnos a la respuesta de estos interrogantes, se alcanzaba asimismo un objetivo subsiguiente, a saber: adaptar un instrumento de análisis categorial válido ya disponible para la comunidad científica de forma tal que facilite también la revisión sistemática de estudios de investigación sobre blended learning atendiendo expresamente a los objetos de trabajo por los que se han preocupado los equipos de investigadores, a cómo se han caracterizado metodológicamente los trabajos y a cuáles han sido los principales resultados alcanzados.

\section{METODOLOGÍA Y FASES DEL ESTUDIO}

La revisión de literatura se realizó en tres fases consecutivas: definición de los criterios de inclusión/exclusión, recogida de datos y análisis de los datos. En un primer momento se desarrolló una revisión teórica de los diferentes conceptos relacionados con nuestros objetivos de investigación: blended learning, formación, desarrollo profesional y liderazgo. Una vez efectuada la definición y establecimiento de criterios que debían cumplir los documentos seleccionados, se realizó la búsqueda de artículos que tuvieran un índice de calidad reconocido por la comunidad científica. Como subraya Pérez-Escoda $\left(2017^{1}\right)$ es importante una alfabetización apropiada que guíe al investigador en la búsqueda, selección y análisis de la información ante la multiplicación de canales, índices, métricas, publicaciones que coexisten en la actualidad.

Por ello, decidimos centrar nuestra búsqueda de documentos en la plataforma de información científica Web Of Science (WOS) de Thomson Reuters (adquirida por Onex Corporation y Baring Private Equity Asia en el 2016), plataforma considerada como la de mayor prestigio entre la comunidad científica. Desde WOS, se accede a las bases de datos del Institute for Scientific Information (ISI) de Thomson Reuters, así como a herramientas de análisis que permiten valorar la calidad científica de las publicaciones a partir de datos objetivos y al alcance de todos. En estas bases de datos se dispone de enlace a textos completos o resúmenes de documentos científicos, recogidos según áreas de conocimiento: «Science Citation Index Expanded» (SCIE), «Social Science Citation Index» (SCCI), «Arts\&Humanities Citation Index» (AHCI) y desde 2015 a «Emerging Sources Citation Index» (ESCI), así como a diferentes herramientas de análisis para valorar tanto la calidad científica de las publicaciones 
como el impacto de la producción científica de un autor y su visibilidad en el mundo académico y científico en general.

En nuestro caso nos centramos en la base de datos principal de «Social Science Citation Index» (SCCI) que recoge la documentación científica relacionada con la investigación en Ciencias Sociales. En ella, centramos la búsqueda teniendo en cuenta:

- Que respondieran a la combinación de los tópicos de nuestro estudio: «blended learning» or «blearning» and «profesional development» or «school principal» or «training»: 190 documentos localizados;

- Que fueran documentos específicamente, artículos de investigación, adscritos a la categoría de investigación educativa «education educational research»: 163 documentos localizados, de los que 75 eran artículos de investigación;

- Que fueran documentos centrados en el desarrollo profesional del docente, excluyendo, por tanto, aquellos relacionados con otras áreas de investigación diferentes a la de investigación educativa, localizando un total de 35 artículos.

Como se observa en la siguiente tabla, de acuerdo con los datos obtenidos a partir de las herramientas de análisis de la propia WOS, los artículos centrados en nuestros temas de estudio tienen fechas de publicación relativamente recientes (ningún documento anterior al 2005) situándose el mayor volumen de publicaciones, en fechas muy recientes (año 2016) lo que indica la cada vez mayor importancia que se le está empezando a conceder.

Tabla 1 y Figura 1. Año de publicación identificado en Web of Science (ISI)

\begin{tabular}{|l|c|c|c|}
\hline Año & F doc. & $\%$ & $\begin{array}{c}\% \\
\text { Acum. }\end{array}$ \\
\hline 2005 & 1 & 3 & 3 \\
\hline 2007 & 1 & 3 & 6 \\
\hline 2008 & 4 & 11 & 15 \\
\hline 2009 & 2 & 6 & 21 \\
\hline 2010 & 1 & 3 & 24 \\
\hline 2011 & 4 & 11 & 37 \\
\hline 2012 & 2 & 6 & 43 \\
\hline 2013 & 1 & 3 & 46 \\
\hline 2014 & 3 & 9 & 55 \\
\hline 2015 & 3 & 9 & 64 \\
\hline 2016 & 12 & 33 & 97 \\
\hline 2017 & 1 & 3 & 100 \\
\hline Total & 35 & $100 \%$ & \\
\hline
\end{tabular}




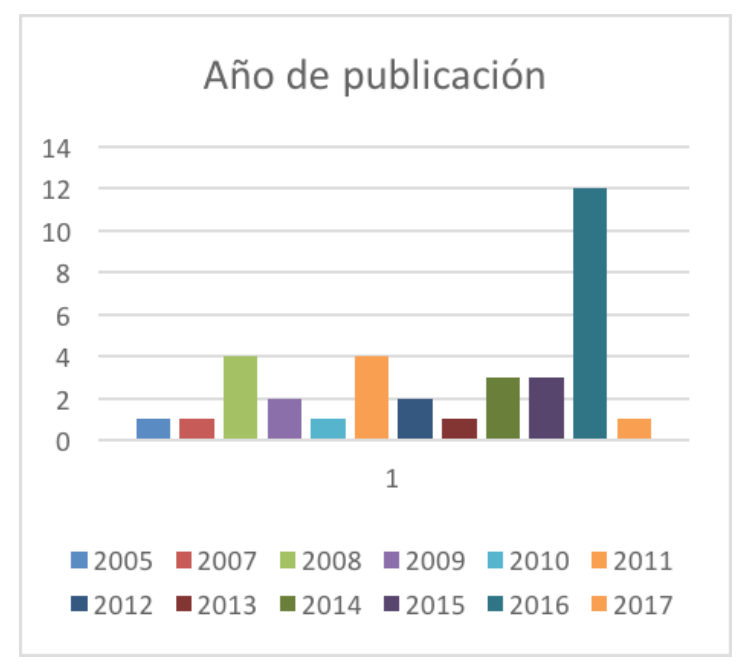

En relación a los países y territorios de procedencia de los autores, como se observa en la siguiente tabla y aunque la mayoría provienen de países anglosajones, nos encontramos con una importante diversidad de países o territorios de origen (21 zonas identificadas), lo que de nuevo nos hace pensar en la importancia creciente que se le está empezando a conceder, en muchas zonas diferentes del mundo.

Tabla 2 y Figura 2. País/región de procedencia de los autores registrados en Web of Science (ISI)

\begin{tabular}{|l|c|c|c|}
\hline Pais/ territorio & F & \% & \% Acum. \\
\hline England & 7 & 20,00 & 20,00 \\
\hline Australia & 6 & 17,14 & 37,14 \\
\hline Canada & 5 & 14,29 & 51,43 \\
\hline Usa & 4 & 11,43 & 62,86 \\
\hline Spain & 3 & 8,57 & 71,43 \\
\hline New Zealand & 2 & 5,71 & 77,14 \\
\hline Uk & 2 & 5,71 & 82,86 \\
\hline Peoples R China & 2 & 5,71 & 88,57 \\
\hline Israel & 1 & 2,86 & 91,43 \\
\hline Venezuela & 1 & 2,86 & 94,29 \\
\hline India & 1 & 2,86 & 97,14 \\
\hline Uruguay & 1 & 2,86 & 100 \\
\hline Total & 35 & $100 \%$ & \\
\hline & \multicolumn{3}{|l}{} \\
\hline
\end{tabular}




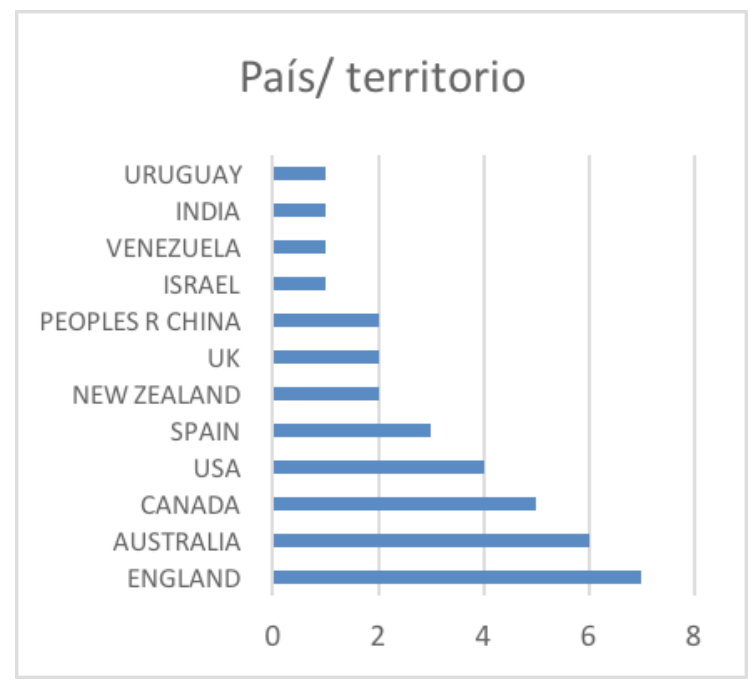

Por último, tras una lectura pormenorizada de cada uno de los documentos, se seleccionaron aquellos que efectivamente se centraban en los tópicos principales de los que partíamos. Condensándose la selección final en 24 artículos:

Tabla 3. Relación de artículos identificados en Web of Science (ISI)

\begin{tabular}{|l|l|l|l|}
\hline F & \multicolumn{1}{|c|}{$\begin{array}{l}\text { Documentos - artículos seleccionados } \\
\text { WOS }\end{array}$} & \multicolumn{1}{|c|}{ Source title: } & Año \\
\hline 1. & $\begin{array}{l}\text { A blended in-service arrangement for } \\
\text { classroom technology integration: impacts on } \\
\text { teachers and students }\end{array}$ & $\begin{array}{l}\text { Computers in Human } \\
\text { Behavior, 21(3), pp. 523-539. }\end{array}$ & 2005 \\
\hline 2. & $\begin{array}{l}\text { Learners' attitudes to wiki technology } \\
\text { in problem based, blended learning for } \\
\text { vocational teacher education }\end{array}$ & $\begin{array}{l}\text { Australasian Journal of } \\
\text { Educational Technology, } \\
\text { 24(4), pp. 425-441. }\end{array}$ & 2008 \\
\hline 3. & $\begin{array}{l}\text { Blended teacher professional development: A } \\
\text { synthesis of three program evaluations }\end{array}$ & $\begin{array}{l}\text { Internet and Higher } \\
\text { Education 11, pp. 201-210. }\end{array}$ & 2008 \\
\hline 4. & $\begin{array}{l}\text { Blended learning For Professional } \\
\text { Development: An Evaluation Of A Program } \\
\text { For Middle School Mathematics And Science } \\
\text { Teachers }\end{array}$ & $\begin{array}{l}\text { Australasian Journal of } \\
\text { Educational Technology, } \\
\text { 27(7), pp. 1124-1134. }\end{array}$ & 2008 \\
\hline 5. & $\begin{array}{l}\text { Professional Development of Physics Teachers } \\
\text { in an Evidence-Based Blended Learning } \\
\text { Program }\end{array}$ & $\begin{array}{l}\text { Journal of Science Education } \\
\text { and Technology, 17(4), pp. } \\
\text { 399-409. }\end{array}$ & 2008 \\
\hline
\end{tabular}




\begin{tabular}{|c|c|c|c|}
\hline $\mathbf{F}$ & $\begin{array}{l}\text { Documentos - artículos seleccionados } \\
\text { WOS }\end{array}$ & Source title: & Año \\
\hline 6. & $\begin{array}{l}\text { Exploring Blended learning for Science } \\
\text { Teacher Professional Development in an } \\
\text { African Context }\end{array}$ & $\begin{array}{l}\text { The International Review } \\
\text { Of Research In Open And } \\
\text { Distributed Learning, 10(4), } \\
\text { pp. 1-19. }\end{array}$ & 2009 \\
\hline 7. & $\begin{array}{l}\text { Toward 'hybridised' faculty development } \\
\text { for the twenty-first century: blending online } \\
\text { communities of practice and face-to-face } \\
\text { meetings in instructional and professional } \\
\text { support programmes }\end{array}$ & $\begin{array}{l}\text { Innovations in Education and } \\
\text { Teaching International, pp. } \\
\text { 261-270. }\end{array}$ & 2010 \\
\hline 8. & $\begin{array}{l}\text { Teacher education from e-learner to } \\
\text { E-teacher: Master curriculum }\end{array}$ & $\begin{array}{l}\text { Turkish Online Journal of } \\
\text { Educational Technology, pp. } \\
\text { 202-212. }\end{array}$ & 2010 \\
\hline 9. & $\begin{array}{l}\text { Knowledge management in blended learning: } \\
\text { Effects on professional development in } \\
\text { creativity instruction }\end{array}$ & $\begin{array}{l}\text { Computers \& Education, } \\
56(1), \text { pp. } 146-156 .\end{array}$ & 2011 \\
\hline 10. & $\begin{array}{l}\text { Interconnecting Networks of Practice for } \\
\text { Professional Learning }\end{array}$ & $\begin{array}{l}\text { International Review of } \\
\text { Research in Open and } \\
\text { Distance Learning, 12(3), pp. } \\
\text { 1-18. }\end{array}$ & 2011 \\
\hline 11. & $\begin{array}{l}\text { Collaborative learning with a wiki: Differences } \\
\text { in perceived usefulness in two contexts of use }\end{array}$ & $\begin{array}{l}\text { Journal of Computer Assisted } \\
\text { Learning, 27(3), pp. 228-242. }\end{array}$ & 2011 \\
\hline 12. & $\begin{array}{l}\text { Preparing teacher leaders: Perceptions of } \\
\text { the impact of a cohort-based, job embedded, } \\
\text { blended teacher leadership program }\end{array}$ & $\begin{array}{l}\text { Teaching and Teacher } \\
\text { Education, 28(4), pp.1213- } \\
1222 .\end{array}$ & 2011 \\
\hline 13. & $\begin{array}{l}\text { Impact of online support for teachers' open- } \\
\text { ended questioning in pre-k science activities }\end{array}$ & $\begin{array}{l}\text { Teaching and Teacher } \\
\text { Education, 28(4), pp. 568-577 }\end{array}$ & 2012 \\
\hline 14. & $\begin{array}{l}\text { A Preliminary Evaluation Of Short Blended } \\
\text { Online Training Workshop For Tpack } \\
\text { Development Using Technology Acceptance } \\
\text { Model }\end{array}$ & $\begin{array}{l}\text { TOJET: The Turkish Online } \\
\text { Journal of Educational } \\
\text { Technology, 11(3), pp. 20-32. }\end{array}$ & 2012 \\
\hline 15. & $\begin{array}{l}\text { Do blended virtual learning communities } \\
\text { enhance teachers' professional development } \\
\text { more than purely virtual ones? A large scale } \\
\text { empirical comparison }\end{array}$ & $\begin{array}{l}\text { Computers \& Education, } \\
60(1), \text { pp.40-51. }\end{array}$ & 2013 \\
\hline 16. & $\begin{array}{l}\text { A perspective on supporting STEM academics } \\
\text { with blended learning at an Australian } \\
\text { university }\end{array}$ & $\begin{array}{l}\text { Issues in Educational } \\
\text { Research, 25(4), pp. 46o-479. }\end{array}$ & 2015 \\
\hline 17. & \begin{tabular}{|l|} 
Open Sim and Sloodle Integration for \\
Preservice Foreign Language Teachers' \\
Continuing Professional Development: \\
A Comparative Analysis of Learning \\
Effectiveness Using the Community of Inquiry \\
Model
\end{tabular} & $\begin{array}{l}\text { Journal of Educational } \\
\text { Computing Research, 54(3), } \\
\text { pp. 1-34. }\end{array}$ & 2015 \\
\hline
\end{tabular}




\begin{tabular}{|c|c|c|c|}
\hline $\mathbf{F}$ & $\begin{array}{c}\text { Documentos - artículos seleccionados } \\
\text { WOS }\end{array}$ & Source title: & Año \\
\hline 18. & $\begin{array}{l}\text { Social presence in the 21st century: An } \\
\text { adjustment to the Community of Inquiry } \\
\text { framework }\end{array}$ & $\begin{array}{l}\text { British Journal of Educational } \\
\text { Technology, 47(6) pp. 1202- } \\
1216 .\end{array}$ & 2016 \\
\hline 19. & $\begin{array}{l}\text { Investigating engagement in a blended } \\
\text { learning course }\end{array}$ & $\begin{array}{l}\text { Cogent Education, 3(1), pp. } \\
1-13 .\end{array}$ & 2016 \\
\hline 20. & $\begin{array}{l}\text { Blending our practice: using online and face- } \\
\text { to-face methods to sustain community among } \\
\text { faculty in an extended length professional } \\
\text { development program }\end{array}$ & $\begin{array}{l}\text { Innovations in Education and } \\
\text { Teaching International 53(6), } \\
\text { pp. 605-615. }\end{array}$ & 2016 \\
\hline 21. & $\begin{array}{l}\text { Cloud Computing Applications And Services } \\
\text { Fostering Teachers' Self- Efficacy }\end{array}$ & $\begin{array}{l}\text { Journal of e-Learning and } \\
\text { Knowledge Society, 12(2), pp. } \\
\text { 85-99. }\end{array}$ & 2016 \\
\hline 22. & $\begin{array}{l}\text { Analysis of an Asynchronous Online } \\
\text { Discussion as a Supportive Model for Peer } \\
\text { Collaboration and Reflection in Teacher } \\
\text { Education }\end{array}$ & $\begin{array}{l}\text { Journal of Information } \\
\text { Technology Education: } \\
\text { Research, 15, pp. 377-401. }\end{array}$ & 2016 \\
\hline 23. & $\begin{array}{l}\text { The educational problem that MOOCs could } \\
\text { solve: professional development for teachers } \\
\text { of disadvantaged students }\end{array}$ & $\begin{array}{l}\text { Research in Learning } \\
\text { Technology, 24(1) pp. 1-24. }\end{array}$ & 2016 \\
\hline 24. & $\begin{array}{l}\text { Design Framework for an Adaptive } \\
\text { MOOC Enhanced by Blended Learning: } \\
\text { Supplementary Training and Personalized } \\
\text { Learning for Teacher Professional } \\
\text { Development }\end{array}$ & $\begin{array}{l}\text { The Electronic Journal of } \\
\text { e-Learning, 14(1), pp.15-30. }\end{array}$ & 2016 \\
\hline
\end{tabular}

Como ya se avanzó, nuestra pretensión respecto de los artículos seleccionados era obtener información sobre cuestiones relacionadas con la identificación del propio documento; la autoría y patrocinio (género, número de autores participantes, apoyo institucional); en relación a los objetivos del estudio; al método, muestra e instrumentos utilizados para la recogida de datos, así como en relación a los resultados que arrojan en términos de eficacia de la modalidad utilizada, y qué condiciones influyen en dicha eficacia.

\section{Procedimiento de codificación y análisis de los datos}

Para responder a las cuestiones planteadas para esta revisión, se editó un cuestionario que combinaba preguntas abiertas y cerradas en GoogleDrive. El cuestionario da continuidad a las investigaciones llevadas a cabo por Cabero et al. (2008) y Tallent-Runnels et al. (2006) y al metaanálisis realizado por Means et al. (2010). Expresamente, el cuestionario responde a una versión adaptada del 
generado por el equipo de investigación liderado por el profesor Cabero y mejorada con la contribución de los otros trabajos citados.

Cabero et al. (2008), siguiendo una metodología Delphi, desarrollaron la «Ficha de análisis de los contenidos de los documentos relacionados con el e-learning y los resultados alcanzados», de la que demostraron ser un instrumento con una alta valía y consistencia. De ella, se han seleccionado solo aquellos interrogantes e ítems que eran útiles para abordar las cuestiones planteadas en nuestro estudio, teniendo en cuenta también como ya se ha señalado la revisión de la literatura llevada a cabo por Tallent-Runnels et al. (2006) sobre la enseñanza y el aprendizaje online. La selección resultante fue ampliada con preguntas adicionales que atendían a las dimensiones consideradas en el metaanálisis realizado por Means et al. (2010) en el marco del estudio que llevan a cabo desde la oficina de planificación, evaluación y desarrollo de políticas educativas del Departamento de Educación de los Estados Unidos para analizar las evidencias basadas en la práctica sobre la efectividad del aprendizaje online en escuelas K-12.

Resultante del cruce de estos estudios, teniendo en cuenta las características específicas de nuestras cuestiones de investigación, generamos así el cuestionario del que nos servimos. Este consta de cinco grandes secciones las cuales pasamos a describir. Véase tabla 4.

Tabla 4. Secciones del instrumento de codificación

\begin{tabular}{|ll|}
\hline 1. & Datos identificativos del documento, autoría y patrocinio \\
\hline 2. & Método, muestra e instrumento \\
\hline 3. & Objeto de investigación \\
\hline 4. & Principales resultados alcanzados \\
\hline 5. & Limitaciones del estudio \\
\hline
\end{tabular}

Se recogieron y analizaron los resultados alcanzados en cada estudio así como las limitaciones reconocidas por los propios autores en el documento, o encontradas durante su lectura.

Como hemos comentado anteriormente, para facilitar la revisión de los documentos finalmente seleccionados, se realizó un formulario online diseñado ad hoc (http://bit.ly/2rB4gba), en el que se cumplimentaron los diferentes aspectos o dimensiones relevantes para el estudio de cada documento. El motivo de efectuar el proceso de análisis de los datos de esta forma, fue la inquietud por realizarlo de manera colaborativa y consensuada, ya que de esta forma las investigadoras tenían acceso tanto al formulario como a los datos introducidos en todos los ítems, desde cualquier lugar y en cualquier momento. Permitiendo así realizar una mayor triangulación del análisis de los datos y conseguir una mayor fiabilidad de los mismos. 


\section{ANÁLISIS DE RESULTADOS}

En relación a los datos identificativos de los documentos, autoría y patrocinio, indicar que se tratan de artículos de investigación publicados en una diversidad de revistas incluidas en la base de datos principal de WOS, en las que se localizan entre uno y dos de los artículos centrados en nuestra temática de estudio, como se observa en la siguiente tabla:

Tabla 5. Título de la revista y año de publicación

\begin{tabular}{|l|l|}
\hline \multicolumn{1}{|c|}{ Source title: } & \multicolumn{1}{c|}{ Año } \\
\hline Australasian Journal of Educational Technology & 2008 \\
\hline British Journal of Educational Technology & 2016 \\
\hline Cogent Education & 2016 \\
\hline Computers \& Education & 2011,2013 \\
\hline Computers in Human Behavior & 2005 \\
\hline $\begin{array}{l}\text { Innovations in Education and Teaching } \\
\text { International }\end{array}$ & 2010,2016 \\
\hline $\begin{array}{l}\text { International Review of Research in Open and } \\
\text { Distance Learning }\end{array}$ & 2011 \\
\hline Internet and Higher Education & 2008 \\
\hline Issues in Educational Research & 2015 \\
\hline Journal of Computer Assisted Learning & 2011 \\
\hline Journal of Educational Computing Research & 2015 \\
\hline Journal of e-Learning and Knowledge Society & 2016 \\
\hline Journal of Information Technology Education & 2016 \\
\hline Journal of Science Education and Technology & 2008 \\
\hline Research in Learning Technology & 2016 \\
\hline Teaching and Teacher Education & 2011,2012 \\
\hline The Electronic Journal of e-Learning & 2016 \\
\hline $\begin{array}{l}\text { The International Review Of Research In Open } \\
\text { And Distributed Learning }\end{array}$ & 2009 \\
\hline $\begin{array}{l}\text { Turkish Online Journal of Educational } \\
\text { Technology }\end{array}$ & 2010 \\
\hline $\begin{array}{l}\text { TOJET: The Turkish Online Journal of } \\
\text { Educational Technology }\end{array}$ & 2010,2012 \\
\hline
\end{tabular}

En cuanto a los autores que firman los diferentes artículos, nos encontramos con una gran mayoría de mujeres: casi la mitad de los documentos fueron firmados por mujeres, 4 de ellos en solitario, otros 7 en colaboración entre dos, tres, e incluso 
más de tres mujeres; y otros 9 en colaboración con algún autor masculino. Solo 4 de los documentos analizados fueron firmados por un hombre en solitario. Estos datos constatan una mayor consideración del tema como centro de interés especialmente para las mujeres, y además, una tendencia importante a realizarlos en colaboración, como es el caso de 16 de los 24 documentos.

Tabla 6. Datos identificativos de autoría

\begin{tabular}{|c|c|c|c|c|c|}
\hline No autores & F & $\%$ & Género & $F$ & $\%$ \\
\hline \multirow{2}{*}{1} & \multirow{2}{*}{8} & \multirow{2}{*}{33} & Hombre & 4 & 17 \\
\hline & & & Mujer & 4 & 17 \\
\hline \multirow{2}{*}{2} & \multirow{2}{*}{6} & \multirow{2}{*}{25} & Mujer & 1 & 4 \\
\hline & & & Mixto & 5 & 20 \\
\hline \multirow{2}{*}{3} & \multirow{2}{*}{6} & \multirow{2}{*}{25} & Mujer & 3 & 12 \\
\hline & & & Mixto & 3 & 13 \\
\hline \multirow{3}{*}{+3} & \multirow{2}{*}{4} & \multirow{2}{*}{17} & Mujer & 3 & 13 \\
\hline & & & Mixto & 1 & 4 \\
\hline & 24 & 100 & & 24 & 100 \\
\hline
\end{tabular}

En referencia a la procedencia de los autores de los artículos, resaltamos que el $100 \%$ de la muestra son personas que mantienen una vinculación profesional con instituciones universitarias. No hemos encontrado documentos de investigación de carácter interuniversitario. En cuanto al apoyo institucional, hemos de indicar que solo en 5 de los 24 artículos se menciona de forma explícita que el estudio tuviera algún tipo de cobertura institucional o subvención a cargo de proyectos $\mathrm{I}+\mathrm{D}$.

En nuestra investigación, pretendíamos conocer también el tipo de estudio que preferentemente se lleva a cabo en los artículos seleccionados, según el grado de manipulación de las variables: estudio de tipo descriptivo (no se manipula ningún tipo de variable, sino que solo se describe y presenta la experiencia formativa), experimental (se manipula una o varias variables independientes, ejerciendo el máximo control), y ex post facto (centrados en analizar eventos ya ocurridos). En este sentido, indicar que en su gran mayoría se tratan de estudios de carácter descriptivo $(\mathrm{f}=18)$ siendo uno de ellos (Brooks, 2010) realizado desde la perspectiva del análisis documental; y otro de carácter autoetnográfico (Hains-Wesson y Tytler, 2015).

Solo hemos encontrado una investigación que responde al tipo ex post facto (Voogt, Almekinders, van den Akker y Moonen, 2005). Por último, indicar que solo 5 de los artículos revisados (21\% de la muestra) describen estudios de carácter experimental. En estos estudios se divide la muestra entre grupo de control y grupo experimental controlando el tipo de metodología seguida: presencial en el caso del grupo de control, y siguiendo un curso blended learning en el caso del grupo 
experimental (Plešec Gasparič, Pečar y Lee, 2016) o realizando discusión online (Kinzie y Whittaker, 2012). Y con pruebas de control de la situación experimental pretest y postest (Yu-Chu, Huang y Yeh, 2011; Oddone, 2016, Pellas y Boumpa, 2015).

De acuerdo a la naturaleza de la información recogida para responder a las cuestiones de investigación, la mayoría de los estudios que hemos indicado como descriptivos, se configuran como estudios de caso, con una metodología mixta en la que se utilizan estrategias e instrumentos de recogida de datos tanto de carácter cualitativo como cuantitativo para obtener una visión más holística de la realidad explorada. Este tipo de estudios se apoya en argumentos, como los esgrimidos por autores como Yin (2009) que consideran el estudio de caso como un método apropiado para investigar "un fenómeno contemporáneo en profundidad y dentro de su contexto real, especialmente cuando los límites entre fenómeno y contexto no son claramente evidentes" (p. 18).

En cuanto a la muestra utilizada, hemos de indicar que nos ha llamado especialmente la atención la falta de especificidad del método de muestreo empleado en los estudios analizados, así como la baja representatividad de las muestras seleccionadas. El método de muestreo, si es que se especifica, responde al tipo no probabilístico e intencional siendo la representatividad de las muestras muy subjetiva.

Del mismo modo que el método de muestreo no es especificado, son pocos los estudios que recogen limitaciones a sus trabajos. Precisamente en aquellos donde aparecen se reconoce que la muestra ha sido muy específica y limitada (Armellini y De Stefani, 2016; Lee, Kinzie y Whittaker, 2012; Robertson, 2008; Tay, 2016).

El análisis del contenido de los resultados alcanzados por cada uno de los trabajos sometidos a revisión nos permite presentar hasta cinco conclusiones que se apoyan, se sostienen, por medio de sus datos. Estas son:

La primera de las conclusiones que se extrae de los documentos analizados, es que el blended learning es reconocido como modalidad de formación óptima para el desarrollo profesional docente. El blended learning es un formato de formación, modo, de formación viable para el desarrollo profesional docente (Owston, Wideman, Murphy y Lupshenyuk, 2008) que cuenta además con gran aceptación (Alsofyani, Aris, Eynon y Majid, 2012). Sea como sea que se desarrolle la formación online (por ejemplo, como curso MOOC) se prefiere la combinación formación presencial y virtual (Gynther, 2016). Esta conclusión se refleja en 17 de los 24 textos analizados, convirtiéndose en la conclusión más ampliamente apoyada.

En segundo lugar, en los diferentes estudios analizados, se observa cómo el blended learning contribuye al desarrollo profesional docente con la consiguiente incidencia en la práctica de enseñanza. Por su parte, un cuarto de los documentos analizados (6, en total) apuntan a que la formación en modalidad blended learning mejora el conocimiento profesional y la eficacia de la enseñanza de los docentes que la cursan (Yeh, Huang y Yeh, 2011), ayudándolos a implementar 
efectivamente estrategias de enseñanza facilitadoras de un aprendizaje significativo (Lee, Kinzie y Whittaker, 2012) y llegando a moverlos hacia innovaciones tal como puede ser la incorporación de las tecnologías en sus prácticas (Voogt, Almekinders, van de Akker y Moonen, 2005). Además, no solo los conocimientos y habilidades de los docentes aumentan sino que se producen transformaciones positivas en la autopercepción de sus competencias (Oddone, 2016) y en la forma cómo los docentes entienden su función (Ross, Adams, Bondy, Dana, Dodman y Swain, 2011).

Una tercera conclusión a la que se llega en los estudios analizados es que el blended learning como modalidad que combina formación a su vez en dos modos diferenciados (presencial + online) requiere de un diseño instruccional global, de conjunto. Si bien la formación blended learning es facilitada en presencial y en virtual, ambos entornos deben jugar diferente pero complementario papel (Berger, Eylon y Bagno, 2008). Al diseñarse se ha de pensar como un todo de forma tal que se combinen coherentemente y con eficiencia las actividades de aprendizaje en línea y presenciales, utilizando las fortalezas de ambas modalidades y proporcionando así una experiencia más provechosa que la que tendría lugar en cualquiera de las dos por separado (Paskevicius y Bortolin, 2016; Tay, 2016). Esta conclusión se recoge en 6 de los 24 textos.

Como cuarta conclusión se observa que en formación blended learning es vital la presencia social. Aunque no se produzca contacto físico entre los participantes de la formación, se precisa de interacción social como requisito para que la experiencia sea gratificante y provechosa. Esto es lo que reconocemos como presencia social y en la literatura sobre e-learning podemos encontrar ampliamente documentado. En la modalidad que nos ocupa, el componente de presencia social se ha mostrado como central, impregnando a la enseñanza misma e incidiendo en la presencia cognitiva (Armellini y De Stefani, 2016). Las implicaciones positivas que se derivan de promover el diálogo en busca de un aprendizaje de carácter sociocultural y constructivista asimismo se han evidenciado (Brooks, 2010). La discusión y la colaboración estimula el crecimiento profesional docente (Plešec y Pečar, 2016). A este respecto, no podemos dudar que el aprendizaje colaborativo se ajusta a todos (Laurillard, 2016) si bien se precisa cuidar el diseño de las actividades que lo fomenten para evitar la tendencia a adoptar un enfoque más cooperativo que de colaboración en su desarrollo (Naismith, Lee y Pilkington, 2011). Además se ha de tomar conciencia de que se puede requerir de inducción en el uso de la tecnología, por ejemplo la wiki, y de ayuda acerca de cómo usarla de manera productiva (Robertson, 2008). Tal es la relevancia de este aspecto, que se invita a plantear la formación de forma que promueva que los participantes formen sus propias redes de práctica dentro y más allá de los parámetros de la experiencia formativa (Mackey y Evans, 2011). Para que así sea, se ha de garantizar la continuidad entre y el sentido de comunidad tanto en la presencialidad como en la virtualidad (Owston, Wideman, Murphy y Lupshenyuk, 2008). Después de la primera de las conclusiones enunciadas, esta es la que aparece 
reflejada en mayor número de documentos. Se cita en la mitad de los sometidos a examen (12, en total).

Por último, no por ello menos importante, de los estudios analizados se muestra la necesidad de que la participación de los docentes en acciones formativas blended learning para el desarrollo profesional tiene que ser facilitada desde las propias instituciones educativas. Desde el entorno, en el ámbito de trabajo, se debe de apoyar la formación y el aprendizaje de los docentes. Especialmente, los docentes encuentran a menudo difícil disponer de tiempo que dedicar después de las horas de trabajo a la formación (Tay, 2016). Los docentes para involucrarse en acciones formativas blended precisan percibir que se reconoce su esfuerzo e interés y se les ayuda (Boitshwarelo, 2009). Aunque la conclusión se recoge en 3 de los 24 textos, en ellos se dedican amplias referencias a la cuestión.

\section{CONCLUSIONES}

En la actualidad, y desde las últimas décadas proliferan trabajos sobre blended learning y desarrollo profesional, constituyendo la alianza de ambas el fin mismo de numerosas investigaciones. Así tenía que ser, tomando en cuenta que la creciente expansión de los entornos abiertos de formación tendría que dar lugar a la creación de campos especializados dentro de ella. Aumentan considerablemente el número de trabajos dedicados a analizar las competencias del desarrollo profesional, los perfiles de liderazgo para canalizar la formación; claro está que las posibilidades de las redes suponen un elemento más de análisis y es en este contexto donde debe realizarse cualquier estudio que se haga sobre ella. Por ello, se ha considerado lo más razonable para comprender la evolución sufrida por estas investigaciones, comenzar por conocer los primeros pasos, hasta llegar al estado actual de la cuestión a nivel tanto internacional como nacional.

La finalidad de nuestro estudio estuvo enfocada desde el inicio a la exploración y análisis de estudios que se refieran a las actividades formativas en modelo blended learning vinculadas al desarrollo profesional y, nuestro interés para con ello ha radicado en ver los objetos de estudio e interrogantes de los equipos que han trabajado en este campo, sus enfoques metodológicos y los resultados más destacables.

Las conclusiones más relevantes quedan agrupadas en cinco grandes grupos referenciados anteriormente: la alta valoración de blended learning como modalidad formativa; su contribución al desarrollo profesional docente y repercusiones a nivel práctico; la necesidad de un diseño instruccional global; la importancia de la presencia social y la necesidad de que sean las instituciones educativas las que impulsen y faciliten la participación de los docentes.

Todo lo anterior nos llevó a resaltar que este modelo de formación puede aportar numerosas posibilidades al desarrollo profesional en todos los componentes de la dimensión social de las organizaciones aglutinados por expertos e investigadores en 
este ámbito tales como la comunicación, la participación, la colaboración, resolución de conflictos, estilos de liderazgo, etc., ya que consideramos que puede eliminar barreras, mejorar la cooperación, aumentar la flexibilidad, facilitar su desarrollo y la interacción entre ellos.

A través de todo lo expuesto, y a la vista de las investigaciones en curso en la actualidad, pensamos que queda mucho camino por recorrer, y mucho por avanzar, y que en un futuro próximo los entornos telemáticos pueden brindar muchas posibilidades que optimicen y enriquezcan los procesos de liderazgo en el ámbito de la formación inicial y permanente.

La reflexión anterior conecta con Owston, Sinclair, Wideman (2008), ya que en su estudio resaltan que el blended learning es un modelo promisorio, aunque hasta ahora poco utilizado, para apoyar el desarrollo profesional de los maestros; a pesar de que es un modelo que combina las ventajas de la interacción cara a cara tradicional con la flexibilidad del aprendizaje en línea.

Nuestro análisis de resultados ha arrojado a este campo de investigación nuevas inquietudes que serán abordadas como futuras líneas de investigación tales como la perspectiva de género en el desarrollo de estos estudios (de acuerdo con los resultados obtenidos en la revisión realizada, prevalece el sexo femenino en la autoría de los mismos), el enfoque colaborativo que aporta la formación telemática (observándose en los resultados, una clara tendencia a la realización de estas investigaciones de forma colaborativa) y las repercusiones a la hora de implementar innovaciones e investigaciones, la importancia de la coordinación y la planificación didáctica en el modelo blended learning, las posibilidades de generar buen clima de trabajo a través de esta modalidad de formación, la influencia de los estilos de liderazgo, etc. Todo ello unido a la evolución de la tecnología y de los nuevos escenarios de formación proyecta un fructífero campo de investigación para estudios e investigaciones en diferentes contextos educativos.

\section{APOYOS}

Este trabajo está avalado por el Proyecto I+D+I, titulado "Competencias mediáticas de la ciudadanía en medios digitales emergentes (smartphones y tablets): prácticas innovadoras y estrategias educomunicativas en contextos múltiples" con clave EDU2015-64015-C3-1-R (MINECO/FEDER), financiado por el Fondo Europeo de Desarrollo Regional (FEDER) y Ministerio de Economía y Competitividad de España.

\section{NOTAS}

1. Pérez-Escoda (2017): «WOS Y SCOPUS: Los grandes aliados de todo investigador». Post Publicado el 9 febrero, 2017 en el blog de «Escuela de autores» de la revista Comunicar (http://bit.ly/2pSC3wh). 


\section{REFERENCIAS BIBLIOGRÁFICAS}

Álvarez, M. (2010). Liderazgo compartido: Buenas prácticas de dirección escolar. Madrid: Wolters Kluwer.

Alsofyani, M. M., Aris, B., Eynon, R., y Majid, N. A. (2012). A preliminary evaluation of short blended online training workshop for TPACK development using technology acceptance model. TOJET: The Turkish Online Journal of Educational Technology, 11(3), 20-32. Recuperado de http://bit.ly/2rllvAZ

Argos, J., y Ezquerra, P. (Eds.) (2013). Liderazgo y educación. Santander: Editorial de la Universidad de Cantabria.

Armellini, A., y De Stefani, M. (2016). Social presence in the 21st century: An adjustment to the Community of Inquiry framework. British Journal of Educational Technology, 47(6), 12021216. doi: 10.1111/bjet.12302.

Berger, H., Eylon, B. S., y Bagno, E. (2008). Professional Development of Physics Teachers in an Evidence-Based Blended Learning Program. Journal of Science Education and Technology, 17(4), 399409. doi: 10.1007/s10956-008-9109-3.

Bernal, A., e Ibarrola, S. (2015). Liderazgo del profesor: objetivo básico de la gestión educativa. Revista Iberoamericana de Educación, 67, 55-70. Recuperado de http://bit.ly/2roB2A9

Bjekic D., Krneta R., y Milosevic D. (2010). Teacher education from e-learner to E-teacher: Master curriculum. TOJET: The Turkish Online Journal of Educational Technology, 2, 202-212. Recuperado de http://bit.ly/2rllvAZ

Boitshwarelo, B. (2009). Exploring Blended Learning for Science Teacher Professional Development in an African Context. The International Review of Research in Open and Distributed Learning, 10(4), 1-19. Recuperado de http://bit.ly/2ryqQl

Bolívar, A., López, J., y Murillo, F. J. (2013). Liderazgo en las instituciones educativas.
Una revisión de líneas de investigación. Revista Fuentes, 14, 15-60. Recuperado de http://bit.ly/2q3Qs8U

Borenstein, M. J., Hedges, L. V., Higgins, J. P. T., y Roths-Tein, H. R. (2009). Introduction to meta-analysis. Chichester, UK: Wiley.

Botella, J., y Gambara, H. (2002). Qué es el meta-análisis. Madrid: Biblioteca Nueva.

Brooks, C. F. (2010). Toward 'hybridised' faculty development for the twenty-first century: blending online communities of practice and face-to-face meetings in instructional and professional support programmes. Innovations in Education and Teaching International, 7(2), 261270. doi:10.1080/14703297.2010.498177.

Cabero, J., y otros (2008). Aportaciones al e-learning: desde la Investigación Educativa. Servicio de publicaciones de la Universidad de Sevilla: GID. Recuperado de http://bit.ly/2qYPyih

Cooper, H. (2010). Research synthesis and meta-analysis: A step-by-step approach. Thousand Oaks, CA: Sage.

CRUE (2016). Las TIC en el Sistema Universitario Español. Madrid: CRUE. Recuperado de http://bit.ly/2p31QUS

Gynther, K. (2016). Design Framework for an Adaptive MOOC Enhanced by Blended Learning: Supplementary Training and Personalized Learning for Teacher Professional Development. The Electronic Journal of e-learning, 14(1), 15-30. Recuperado de http://bit.ly/2puSP4q

Hains-Wesson, R., y Tytler, R. (2015). A perspective on supporting STEM academics with blended learning at an Australian university. Issues in Educational Research, 25(4), 460-479. Recuperado de http://bit.ly/2rydIgu

Johnson, L., Adams Becker, S., Cummins, M., Estrada, V., Freeman, A., y Hall, C. (2016). NMC Horizon Report: 2016 
Higher Education Edition. Austin, Texas: The New Media Consortium.

Laurillard, D. (2016). The educational problem that MOOCs could solve: professional development for teachers of disadvantaged students. Research in Learning Technology, 24(1) 1-24. doi: 10.3402/rlt.v24.29369.

Lee, Y., Kinzie, M. B., y Whittaker, J. V. (2012). Impact of online support for teachers' open-ended questioning in pre-k science activities. Teaching and Teacher Education, 28(4), 568-577. doi: 10.1016/j. tate.2012.01.002.

Leithwood, K., y Louis, K. S. (Eds.) (2011). Linking leadership to student learning. San Francisco: Jossey-Bass.

Mackey, J., y Evans, T. (2011). Interconnecting Networks of Practice for Professional Learning. International Review of Research in Open and Distance Learning, 12 (3), 1-18. doi: 10.19173/ irrodl.v12i3.873.

Martín, J., Tobías, A., y Seoane, T. (Coords.) (2006). Revisiones sistemáticas. Toledo: FISCAM.

Martínez Carazo, P. C. (2006). Método de estudio de caso: estrategia metodológica de la investigación científica. Revista Pensamiento \& Gestión, 20, 165-193. Recuperado de http://bit.ly/2q2Yaz

Matzat, U. (2013). Do blended virtual learning communities enhance teachers' professional development more than purely virtual ones? A large scale empirical comparison. Computers $y$ Education, 6o(1), 40-51. doi: 10.1016/j. compedu.2012.08.006.

Means, B., Toyama, Y., Murphy, R., Bakia, M., y Jones, K. (2010). Evaluation of Evidence-Based Practices in Online Learning: A Meta-Analysis and Review of Online Learning Studies. Washington, D.C.: U.S. Department of Education, Office of Planning, Evaluation, and Policy Development. Recuperado de http://bit. ly/1yvyk4P
Modeef, M., Bin Aris, B., Eynon, R., y Abdul, N. (2012). A Preliminary Evaluation of Short Blended Online Training Workshop for Tpack Development Using Technology Acceptance Model. TOJET: The Turkish Online Journal of Educational Technoloqu, 11(3), 20-32. Recuperado de http://bit.ly/2rllvAZ

Naismith, L., Lee, B.-H., y Pilkington, R. M. (2011). Collaborative learning with a wiki: Differences in perceived usefulness in two contexts of use. Journal of Computer Assisted Learning, 27(3), 228-242. doi: 10.1111/j.1365-2729.2010.00393.x.

Oddone, F. (2016). Cloud Computing Applications and Services Fostering Teachers' Self- Efficacy. Journal of $e$-Learning and Knowledge Society, 12(2), 85-99. Recuperado de http://bit. y/2q36SoJ

Owston, R. D., Wideman, H., Murphy, J., y Lupshenyuk, D (2008). Blended teacher professional development: A synthesis of three program evaluations. Internet and Higher Education, 11, 201-210. doi: 10.1016/j.iheduc.2008.07.003.

Owston, R. D., Sinclair, M., y Wideman, H. (2008). Blended Learning For Professional Development: An Evaluation Of A Program For Middle School Mathematics And Science Teachers. Australasian Journal of Educational Technology, 27(7), 1124-1134. Recuperado de http:// www.yorku.ca/rowston/TCRfinal.pdf

Paskevicius, M., y Bortolin, K. (2016). Blending our practice: using online and face-to-face methods to sustain community among faculty in an extended length professional development program. Review Innovations in Education and Teaching International, 53(6), 605-615. doi: 10.1080/14703297.2015.1095646.

Pellas, N., y Boumpa, A. (2015). Open Sim and Sloodle Integration for Preservice Foreign Language Teachers' Continuing Professional Development: A Comparative Analysis of Learning 
Effectiveness Using the Community of Inquiry Model. Journal of Educational Computing Research, 54(3), 1-34. doi: 10.1177/0735633115615589.

Plešec Gasparič, R., y Pečar, M. (2016). Analysis of an Asynchronous Online Discussion as a Supportive Model for Peer Collaboration and Reflection in Teacher Education. Journal of Information Technology Education: Research, 15, 377-401. Recuperado de http://bit. y/2q03FzH

Robertson, I. (2008). Learners' attitudes to wiki technology in problem based, blended learning for vocational teacher education. Australasian Journal of Educational Technologu, 24(4), 425-441. doi: https://doi.org/10.14742/ajet.1202

Ross, D., Adams, A., Bondy, E., Dana, N., Dodman, S., y Swain, C. (2011). Preparing teacher leaders: Perceptions of the impact of a cohort-based, job embedded, blended teacher leadership program. Teaching and Teacher Education, 28(4), 12131222. doi: 10.1016/j.tate.2011.06.005.

Sánchez Meca, J. (2008). Meta-análisis de la investigación. En M.A. Verdugo, M. Crespo, M. Badía y B. Arias (Coords.), Metodología en la investigación sobre discapacidad. Salamanca: Publicaciones del INICO, 121-140. Recuperado de http://bit.ly/2qzx2vQ

Sánchez Meca, J., y Botella, J. (2010). Revisiones sistemáticas y meta-análisis: Herramientas para la práctica profesional. Papeles del Psicólogo, 31(1), 7-17. Recuperado de http://bit.ly/2qCQSEt

Tallent-Runnels, M. K., y otros (2006). Teaching Courses Online: A Review of the Research, Review of Educational Research, 76(1), 93-135. doi: 10.3102/00346543076001093.

Tay, H. Y. (2016). Investigating engagement in a blended learning course. Cogent Education, 3(1), 1-13. doi: 10.1080/2331186X.2015.1135772.

Voogt, J., Almekinders, M., Van den Akker, J., y Moonen, B. (2005). A blended in-service arrangement for classroom technology integration: impacts on teachers and students. Computers in Human Behavior, 21(3), 523-539. doi: 10.1016/j.chb.2004.10.003.

Yeh, Y., Huang, L., y Yeh, Y. (2012). Knowledge management in blended learning: Effects on professional development in creativity instruction. Computers \& Education, 56(1), 146-156. doi: 10.1016/j.compedu.2010.08.011.

\section{PERFIL ACADÉMICO Y PROFESIONAL DE LAS AUTORAS}

Ana Duarte Hueros. Doctora en Psicopedagogía. Profesora Titular de la Universidad de Huelva desde 2001. Imparte docencia en asignaturas de Grado y Máster relacionadas con la tecnología educativa y educomunicación. Ha participado en numerosas investigaciones y publicaciones sobre la temática. Compagina su labor docente e investigadora con la gestión de la Dirección de Enseñanza Virtual de la Universidad de Huelva. Actualmente es miembro activo del Grupo de Investigación «@gora» de la Universidad de Huelva.

E-mail: duarte@uhu.es 
María Dolores Guzmán Franco. Doctora en Psicopedagogía y Profesora Titular del Departamento de Educación de la Universidad de Huelva. Cuenta con casi dos décadas de experiencia docente en materias adscritas al área de Didáctica y Organización en las Titulaciones de Maestro, Psicopedagogía, Educación Social, Posgrados varios y Doctorado. Sus líneas de docencia e investigación están vinculadas a la integración de TIC en el contexto educativo, la formación del profesorado en espacios educativos virtuales y los estudios en competencia mediática.

E-mail: maria.guzman@dedu.uhu.es

\section{DIRECCIÓN DE LAS AUTORAS}

Facultad de Ciencias de la Educación

Universidad de Huelva

Avda. de las Fuerzas Armadas, s/n

21007 Huelva (España)

Carmen Yot Domínguez. Licenciada en Pedagogía por la Universidad de Sevilla en el año 2008. Dispone de la Certificación del Periodo de Docencia del Tercer Ciclo, el Título Diploma de Estudios Avanzados y el Título de Doctor con Mención Internacional. Actualmente profesora asociada de la Universidad Internacional de La Rioja. Miembro del grupo de investigación I.D.E.A. (INNOVACION, DESARROLLO, EVALUACION Y ASESORAMIENTO [referencia HUM423] de la Universidad de Sevilla.

E-mail: carmenyot@us.es

\section{DIRECCIÓN DE LA AUTORA}

Facultad de Ciencias de la Educación

Universidad de Sevilla

$\mathrm{C} /$ Pirotecnia $\mathrm{s} / \mathrm{n}$

41013 Sevilla

Fecha de recepción del artículo: 20/05/2017

Fecha de aceptación del artículo: 02/07/2017

\section{Como citar este artículo:}

Duarte Hueros, A., Guzmán Franco, M. D., y Yot Domínguez, C. R. (2018). Aportaciones de la formación blended learning al desarrollo profesional docente. RIED. Revista Iberoamericana de Educación a Distancia, 21(1), pp. 155-174. doi: http://dx.doi.org/10.5944/ried.21.1.19013 